\title{
Clinical Predictors of COVID-19 Mortality Among Patients in Intensive Care Units: A Retrospective Study
}

\author{
Abbas Al Mutair (D) ${ }^{1-3}$ \\ Alya Al Mutairi ${ }^{4}$ \\ Abdul Rehman Zia Zaidi (iD ${ }^{5,6}$ \\ Samer Salih ${ }^{5}$ \\ Saad Alhumaid (iD) ${ }^{7}$ \\ Ali A Rabaan $\mathbb{1 D}^{8}$ \\ Awad Al-Omari ${ }^{5,6}$
}

'Research Center, Almoosa Specialist Hospital, Al-Ahsa, 31982, Saudi Arabia;

${ }^{2}$ Research Center, College of Nursing, Princess Norah Bint Abdulrahman University, Riyadh, Saudi Arabia; ${ }^{3}$ School of Nursing, University of Wollongong, Wollongong, Australia; ${ }^{4}$ Department of Mathematics, Faculty of Science, Taibah University, Medina, 54321, Saudi Arabia;

${ }^{5}$ Research Center, Dr. Sulaiman Al Habib Medical Group, Riyadh, 12214, Saudi Arabia; ${ }^{6}$ College of Medicine, Alfaisal University, Riyadh, Saudi Arabia;

${ }^{7}$ Administration of Pharmaceutical Care, Al-Ahsa Health Cluster, Ministry of Health, Al-Ahsa, Saudi Arabia; ${ }^{8}$ Molecular Diagnostic Laboratory, Johns Hopkins Aramco Healthcare, Dhahran, Saudi Arabia
Correspondence: Alya Al Mutairi

Email amutairi@taibahu.edu.sa
Objective: The ongoing pandemic of the coronavirus disease 2019 (COVID-19), which originated from Wuhan, China, has been identified to be caused by the novel beta coronavirus, severe acute respiratory syndrome coronavirus 2 (SARS-CoV-2). COVID-19 has been spreading rapidly worldwide within just a few months. Our aims were to analyze clinical and laboratory abnormalities in ICU patients with COVID-19, in order to define which predictors can distinguish between those who are at higher risk of developing fatal versus non-fatal forms of the disease.

Methods: A descriptive cross-sectional survey was used; demographics, comorbidities, symptoms, laboratory parameters at ICU admission, and clinical outcomes for the adult patients admitted to ICU were collected from five hospitals in Saudi Arabia.

Results: A total of 86 patients with COVID-19 admitted in ICU, 50 patients died, 23 recovered, and 13 were still admitted, with a mortality rate of $58.1 \%$. Septic shock (OR (95\% CI): 58.1 (5.97-7812.8), p < 0.001) and acute kidney injury (AKI) (OR (95\% CI): 7.279 (1.191-65.43), $\mathrm{p}=0.032$ ) had a significant impact on mortality. Cox proportionalhazards regression analysis revealed that septic shock (HR (95\% CI): 9.502 (2.958-30.524), $\mathrm{p}<0.001)$ and neutrophil count (HR (95\% CI): 1.053 (1.023-1.085), p < 0.001) were significant predictors for mortality.

Conclusion: Septic shock, AKI, and high neutrophil count were found to be predictive of death in these patients. Further studies are needed to aid efficient recognition and management of severe COVID-19 patients in our population.

Keywords: COVID-19, severe acute respiratory syndrome coronavirus 2, intensive care units, Saudi Arabia, mortality

\section{Introduction}

COVID-19 confirmed patients admitted to ICU and associated mortality is soaring worldwide. ${ }^{1}$ According to the World Health Organization (WHO), the cumulative total of identified COVID-19 positive cases has reached above 34 million with more than $1,000,000$ deaths till October 2, 2020. ${ }^{2}$ The main transmission route of SARS-CoV-2 is through the air (airborne transmission) and on a daily basis the number of deaths associated with COVID-19 is rapidly increasing. ${ }^{3}$ In Saudi Arabia, the first case of COVID-19 was reported on March 02, 2020, in a Saudi national returning from Iran. ${ }^{4}$ To date, there have been over 435,027 confirmed cases of COVID-19 with approximately 7188 deaths in the Kingdom. ${ }^{5}$ Data from the eastern Mediterranean region on COVID-19 is scarce, especially from Saudi Arabia. Take, for instance, the proportion 
of COVID-19 patients admitted to ICU, the clinical course, and the mortality and its predictors in critically ill patients have not been fully investigated. ${ }^{6,7}$ Around $32 \%$ of COVID19 inpatients were admitted to the ICUs in Wuhan, China, and mortality rates of ICU patients differed across provinces. ${ }^{8,9}$ The mainstay treatment of severe and critically COVID-19 ill patients occurs in the ICU where they are monitored, given life support, and treated to reduce mortality. However, during the COVID-19 pandemic, overcrowding of the ICUs, overload and increased burden on healthcare providers have been observed. This led Saudi officials to consider planning expansion of the ICUs, allocating more funding for ICUs, and increasing the number of specialized medical staff. Telemedicine has gained momentum over the past several months as Tele-ICUs opened the door to more accessible and effective care. ${ }^{10}$

Our aims in the current study are to analyze clinical and laboratory abnormalities in ICU patients with COVID19 , in order to define which predictors can distinguish between those who are at higher risk of developing fatal versus non-fatal forms of the disease. This is significant as recognizing the fatal forms of COVID-19 based on the clinical and laboratory predictors is urgently needed. These predictive variables can allow risk stratification, guide interventional studies to target patients at enhanced risk of developing death, and optimize the allocation of limited human and technical resources. Moreover, identification of laboratory parameters capable of differentiating between fatal and non-fatal cases, or those at high or low risk of mortality, will allow for improved clinical situational awareness.

\section{Materials and Methods}

Data of 86 patients were collected from April 282020 to June 302020 from five private tertiary care hospitals from Dr Sulaiman Al Habib Medical Group (HMG). Clinical data were collected after reviewing the patients' electronic medical records, nursing records, clinical findings, laboratory findings, management details, and outcome information. Clinical data at hospital admission, including demographic information, chronic comorbidities, vital signs, symptoms, laboratory tests, and outcomes were collected from the patients' electronic medical records. Epidemiological, clinical, and laboratory characteristics and treatment and outcomes data were obtained using standard collection forms. Two researchers were assigned to double-check the data collected. In this paper, neutrophil and lymphocyte counts are absolute neutrophil and absolute lymphocyte counts. This study received ethical approval from the Institutional Review Board of Dr Sulaiman Al Habib Medical Group (IRB Log No. RC20.06.88-1). As this was a retrospective study, no informed consent was deemed necessary, and data were deidentified for the use of this publication. This study adhered to the ethical guidelines of the Declaration of Helsinki and good clinical practice.

The Saudi Ministry of Health ICU admission and discharge criteria during COVID-19 was utilized as a guideline to recruit our sample in the current study. ${ }^{11}$ As per the guideline of the ICU admission criteria that patient with confirmed SARS-CoV-2 infection and:

1. With unstable vital signs.

2. With hemodynamic instability.

3. Requires invasive or non-invasive mechanical ventilation.

4. Has respiratory distress.

5. Has more than one failure organ.

6. Requires Continuous Renal Replacement Therapy CRRT.

7. Requires vasopressor support.

8. Has decreased level of consciousness

9. With abnormal ECG findings, including arrhythmias, heart blocks and ischemia and

10. Has acidosis: $\mathrm{ABG}$ with $\mathrm{pH}<7.3$ or $\mathrm{PCO} 2>50$ or above patient's baseline.

In the statistical analysis of the current study, we divided the patients into two groups, survivors and non-survivors. Data were analyzed using Statistical Package for the Social Sciences (SPSS) version 24.0 and R version 4.02 by the R Foundation for Statistical Computing. A value of $\mathrm{p}<0.05$ was considered statistically significant. The distribution of the continuous variables was carried out by the Shapiro-Wilk test (survivor versus non-survivor). Frequencies and percentages were calculated for categorical variables. Baseline characteristics were presented as mean \pm standard deviation (SD) and median with interquartile range (IQR) for continuous variables and as numbers with percentages for categorical variables. MannWhitney $U$-test was done where indicated in the tables. Spearman correlation was the main test as most of the inflammatory markers were not normally distributed (violation of Pearson's correlation assumption). Only the pairs with a significant correlation $(p<0.05)$ were presented. And the interpretation of strength was based on the 
guidelines for biostatistics published by Chan in $2003{ }^{8}$ The Chi-square $\left(\chi^{2}\right)$ test was applied to examine categorical data. A $\chi^{2}$ test was also employed to detect the relationship between the categorical variables. The significant differences were used in binary logistic regression to get the odd ratio (OR). We then used survival analysis Cox regression and generated the nomogram for the probability. For the significant factors/predictors, we created the survival probability nomograms. Firth's logistic regression with penalized maximum likelihood was employed for biased estimation due to potential small sample size in subgroups. This approach was developed to reduce the biasness of small sample size, which resulted in finite and consistent estimates even in the case of separation. ${ }^{9-11}$

\section{Results}

In this study, we extracted retrospective data of 86 critically ill adult patients admitted in ICU with laboratoryconfirmed COVID-19 from five Saudi hospitals through April 28 to June 30, 2020. At the cut-off date of data analysis, 50 patients died, 23 recovered, and 13 were still admitted, resulting in a mortality rate as high as $58.1 \%$ for
COVID-19 patients in ICU. It is important to state that 13 censored data due to the end of the data collection timeline were excluded from the analysis of logistic regression. Table 1, presents the baseline characteristics and symptoms of ICU patients on admission, grouped by survivors, and non-survivors. Non-survivors were significantly older than survivors of COVID-19 in ICU (mean age: 58 vs 49 years; $p=0.016$ ). Results also revealed that survivors had higher cough symptoms as compared to non-survivors of COVID-19 in ICU (\% cough: $100 \%$ vs $78 \%$; $p=0.015$ ).

In addition, Table 2 summarizes the baseline comorbidities and complications of survivors and non-survivors admitted in ICU with laboratory confirmed COVID-19 infection. For all types of comorbidities considered, no significant difference was found between survivors and non-survivors of COVID-19 admitted in ICU. Nonetheless, results demonstrated that there is a significantly higher proportion of patients with septic shock complications for non-survivors compared to survivors of COVID-19 in ICU (\% Septic Shock: $84 \%$ vs $0 \%$; p < 0.001). Furthermore, results revealed that there are a significantly higher proportion of patients with acute

Table I Baseline Characteristics and Symptoms of COVID-19 Patients Admitted in ICU

\begin{tabular}{|c|c|c|c|c|c|}
\hline Variables & All $(n=73)$ & Survivors $(n=23)$ & Non-Survivors $(n=50)$ & p value & Test \\
\hline \multicolumn{6}{|l|}{ Demographic } \\
\hline Age & $55(52-59)$ & $49(42-56)$ & $58(54-63)$ & 0.016 & $\mathrm{t}$ \\
\hline Gender (Male) & $65(89)$ & $22(95.7)$ & $43(86)$ & 0.220 & $\chi^{2}$ \\
\hline Healthcare Worker (Yes) & I (I.4) & I (4.3) & $0(0)$ & 0.138 & $\chi^{2}$ \\
\hline Travel History (No) & $64(87.7)$ & $19(82.6)$ & $45(90)$ & 0.372 & $\chi^{2}$ \\
\hline \multicolumn{6}{|l|}{ Physical Examination } \\
\hline Systolic Blood Pressure & $127(122-131)$ & $126(|20-13|)$ & $127(|2|-\mid 33)$ & 0.713 & $\mathrm{t}$ \\
\hline Diastolic Blood Pressure & 7I (68-73) & $72(67-77)$ & $70(66-73)$ & 0.388 & $\mathrm{t}$ \\
\hline Pulse & 93 (89-97) & $95(88-102)$ & $93(88-98)$ & 0.539 & $\mathrm{t}$ \\
\hline Oxygen Saturation & $89(83-94)$ & $89(85-94)$ & $88(8 I-94)$ & 0.552 & $\mathrm{~m}-\mathrm{w}$ \\
\hline Respiratory Rate & $24(22-28)$ & $25(22-28)$ & $24(22-28)$ & 0.188 & $m-w$ \\
\hline \multicolumn{6}{|l|}{ Symptoms } \\
\hline Temperature & $37.8(37.2-38.2)$ & $37.7(37.2-38.1)$ & $37.9(37.1-38.2)$ & 0.775 & $\mathrm{~m}-\mathrm{w}$ \\
\hline Cough (Yes) & $62(84.9)$ & $23(100)$ & $39(78)$ & 0.015 & $\chi^{2}$ \\
\hline Hemoptysis (Yes) & $2(2.7)$ & I (4.3) & $I(2)$ & 0.568 & $\chi^{2}$ \\
\hline Shortness of Breath (Yes) & $62(84.9)$ & $20(87)$ & $42(84)$ & 0.743 & $\chi^{2}$ \\
\hline Runny Nose (Yes) & $3(4.1)$ & $0(0)$ & $3(6)$ & 0.230 & $\chi^{2}$ \\
\hline Nasal Congestion (Yes) & $2(2.7)$ & $0(0)$ & $2(4)$ & 0.331 & $\chi^{2}$ \\
\hline Sore Throat (Yes) & $18(24.7)$ & $6(26.1)$ & $12(24)$ & 0.848 & $\chi^{2}$ \\
\hline GIT symptoms (Yes) & $9(12.3)$ & $2(8.7)$ & $7(14)$ & 0.522 & $\chi^{2}$ \\
\hline Headache (Yes) & $7(9.6)$ & $2(8.7)$ & $5(10)$ & 0.860 & $\chi^{2}$ \\
\hline
\end{tabular}

Notes: $\chi^{2}=$ Chi-square test, $\mathrm{m}-\mathrm{w}=$ Mann-Whitney test, $\mathrm{t}=$ Student's $t$-test. Data were expressed as count $(\%)$ unless otherwise and a $\mathrm{p}$-value of $<0.05$ was considered significant.

Abbreviation: GIT, gastrointestinal tract. 
Table 2 Baseline Comorbidities and Complications of COVID 19 Patients Admitted in ICU

\begin{tabular}{|c|c|c|c|c|c|}
\hline Variables & All $(n=73)$ & Survivors $(n=23)$ & Non-Survivors $(n=50)$ & p value & Test \\
\hline \multicolumn{6}{|l|}{ Comorbidities } \\
\hline Hypertension (Yes) & $22(30.1)$ & $4(17.4)$ & $18(36)$ & 0.107 & $\chi^{2}$ \\
\hline Diabetes (Yes) & $32(43.8)$ & $8(34.8)$ & $24(48)$ & 0.290 & $\chi^{2}$ \\
\hline Cardiovascular Disease (Yes) & $11(15.1)$ & $2(8.7)$ & $9(18)$ & 0.302 & $\chi^{2}$ \\
\hline Lung Disease (Yes) & $5(6.8)$ & $2(8.7)$ & $3(6)$ & 0.672 & $\chi^{2}$ \\
\hline Cerebrovascular Disease (Yes) & $2(2.7)$ & $0(0)$ & $2(4)$ & 0.331 & $\chi^{2}$ \\
\hline Kidney Disease (Yes) & $\mathrm{I}(\mathrm{I} .4)$ & $0(0)$ & I (2) & 0.495 & $\chi^{2}$ \\
\hline Malignancy (Yes) & $\mathrm{I}(\mathrm{l} .4)$ & I (4.3) & $0(0)$ & 0.138 & $\chi^{2}$ \\
\hline Liver Disease (Yes) & $\mathrm{I}(\mathrm{l} .4)$ & $0(0)$ & I (2) & 0.495 & $\chi^{2}$ \\
\hline Autoimmune (Yes) & I (I.4) & I (4.3) & $0(0)$ & 0.138 & $\chi^{2}$ \\
\hline \multicolumn{6}{|l|}{ Complications } \\
\hline Septic Shock (Yes) & $42(57.5)$ & $0(0)$ & $42(84)$ & $<0.001$ & $\chi^{2}$ \\
\hline Pneumonia (Yes) & $73(100)$ & $23(100)$ & $50(100)$ & 1.000 & $\chi^{2}$ \\
\hline ARDS (Yes) & $63(86.3)$ & $15(65.2)$ & $48(96)$ & $<0.001$ & $\chi^{2}$ \\
\hline AKI (Yes) & $4 \mid(56.2)$ & $4(17.4)$ & $37(74)$ & $<0.001$ & $\chi^{2}$ \\
\hline Cardiac Injury (Yes) & $3(4.1)$ & I (4.3) & $2(4)$ & 0.945 & $\chi^{2}$ \\
\hline DIC (Yes) & $2(2.7)$ & $0(0)$ & $2(4)$ & 0.331 & $\chi^{2}$ \\
\hline
\end{tabular}

Notes: Data were expressed as count (\%) unless otherwise and a p-value of $<0.05$ was considered significant.

Abbreviations: ARDS, acute respiratory distress syndrome; AKI, acute kidney injury; DIC, disseminated intravascular coagulation.

respiratory distress syndrome (ARDS) complication among non-survivors as compared to survivors of COVID-19 in ICU (\% ARDS: $65.2 \%$ vs 90\%; p < 0.001). Moreover, we noticed that the proportion of COVID-19 patients admitted in ICU with acute kidney injury (AKI) complication are significantly higher in non-survivors when compared to survivors (\% AKI: $74 \%$ vs $17.4 \%, \mathrm{p}<0.001)$.

Next, we analyzed and summarized the baseline laboratory findings of survivors and non-survivors admitted in ICU with laboratory confirmed COVID-19 infection, as presented in Table 3. From the results, most of the markers do not show a significant difference between survivor and non-survivor of COVID-19 patients in ICU at 0.05 significance level, except for Neutrophil count, D-dimer, and blood urea nitrogen (BUN).

The study examined the correlation between laboratory findings using Spearman correlation and the results were summarized as in Table 4. Spearman correlation was employed as almost all of the variables from laboratory findings showed a significant violation of normality assumption. Using the strength guideline by Chan, ${ }^{8}$ WBC count vs neutrophil count, neutrophil count vs D-dimer, neutrophil count vs NLR Ratio, ALT vs AST, and Creatinine vs BUN have a significant moderate correlation.
However, Table 5 summarized the univariate (unadjusted) and multivariate (adjusted) Firth's logistic regression for predictors/factors of COVID-19 patient's mortality in ICU. From the results, all factors showed a significant impact on the mortality of COVID-19 patients in ICU. Control factor - gender is the only factor that did not indicate a significant relationship to the mortality of the study sample. After controlling demographic covariates and factors/predictors of interest, clearly only septic shock complication (OR (95\% CI): 58.1 (5.97-7812.8), p $<0.001$ ) and AKI complication (OR (95\% CI): 7.279 (1.191-65.43), $\mathrm{p}=0.032$ ) have significant impact on the mortality of COVID-19 patients admitted in ICU. The OR of 58.1 for septic shock means that the odd to die for COVID-19 patients with septic shock complication when admitted to ICU is 58.1 times higher than those who do not exhibit septic shock complication. Then, the OR of 7.279 for AKI indicates that the odd for COVID-19 patients with AKI complication to die when they were admitted to ICU is about 7.3 times higher than those who do not have AKI complication.

Moreover, Table 6 summarizes the univariate (unadjusted) and multivariate (adjusted) Cox proportional hazards regression for predictors/factors of COVID-19 patient's mortality in ICU. After adjusting for all the demographic as control variable, and predictors/ factors 
Table 3 Baseline Laboratory Findings of COVID 19 Patients Admitted in ICU

\begin{tabular}{|c|c|c|c|c|c|}
\hline Variables & All $(n=73)$ & Survivors $(n=23)$ & Non-Survivors $(n=50)$ & p value & Test \\
\hline \multicolumn{6}{|l|}{ Laboratory Findings } \\
\hline WBC count & $8.7(5.8-11.4)$ & $6.9(5.6-11.4)$ & $9.7(5.8-11.7)$ & 0.204 & $m-w$ \\
\hline Hemoglobin & $13.5(13.1-13.9)$ & $13.8(\mid 3-14.5)$ & $13.4(13-13.8)$ & 0.380 & $t$ test \\
\hline Neutrophil count & $7.5(4.7-10.9)$ & $6(3.7-8.8)$ & $9.2(5.1-16.7)$ & 0.011 & $m-w$ \\
\hline Lymphocyte count & $1.26(0.7-1.8)$ & I.I (0.6-I.44) & I.3 (0.7I-2.3) & 0.275 & $\mathrm{~m}-\mathrm{w}$ \\
\hline Platelet & $218(197-239)$ & $210(180-240)$ & 221 (193-249) & 0.624 & $t$ test \\
\hline D-dimer & $1.3(0.8-2.18)$ & $1.16(0.46-1.35)$ & $1.51(0.85-3.25)$ & 0.003 & $\mathrm{~m}-\mathrm{w}$ \\
\hline CRP & I56.I (135.6-176.6) & $153.2(\mid 14.1-192.4)$ & $157.4(132.6-182.3)$ & 0.851 & $t$ test \\
\hline $\mathrm{LDH}$ & $535(4 \mid 8-707)$ & $535(353-724)$ & $535(4 \mid 8-707)$ & 0.722 & $m-w$ \\
\hline ALT & $37(25-53)$ & $31(23-45)$ & $38.5(28-59)$ & 0.077 & $\mathrm{~m}-\mathrm{w}$ \\
\hline AST & $53(34-72)$ & $53(28-6 I)$ & $53.5(34-75)$ & 0.419 & $m-w$ \\
\hline Total bilirubin & $10.1(7-14)$ & II (7-13.5) & $10(7.8-15)$ & 0.894 & $m-w$ \\
\hline BUN & $5.6(3.7-7.9)$ & $4.4(3.1-6.1)$ & $6.1(3.8-8.9)$ & 0.026 & $\mathrm{~m}-\mathrm{w}$ \\
\hline Creatinine & $82.7(66.4-114)$ & $73(64-94)$ & $87.4(71-129)$ & 0.060 & $\mathrm{~m}-\mathrm{w}$ \\
\hline Ferritin & 1378 (794-1970) & $1317(47 \mid-1549)$ & I 437 (88I-240I) & 0.363 & $m-w$ \\
\hline NLR Ratio & $6.2(3.5-12)$ & $5.4(3.8-9.1)$ & $7.1(3.5-14.4)$ & 0.291 & $m-w$ \\
\hline Blood culture (Yes) & $8(\mathrm{II})$ & $0(0)$ & $8(16)$ & 0.042 & $\times 2$ \\
\hline
\end{tabular}

Notes: Data were expressed as count (\%) unless otherwise and a p-value of $<0.05$ was considered significant.

Abbreviations: WBC, white blood cell; CRP, C-reactive protein; LDH, lactate dehydrogenase; ALT, alanine transaminase; AST, aspartate transaminase; BUN, blood urea nitrogen; NLR, neutrophil-lymphocyte ratio.

of interest, septic shock (HR (95\% CI): 9.502 (2.958$30.524), \mathrm{p}<0.001)$ and neutrophil count (HR $(95 \% \mathrm{CI})$ : $1.053(1.023-1.085), \mathrm{p}<0.001)$ were found to be significant predictor for mortality among COVID-19 patients in ICU. For septic shock, the HR of 9.5 indicates that the expected hazard (death) of COVID-19 patients in ICU with such complication is 9.5 times than those who do not have. On the other hand, the HR value of 1.053 for neutrophil count implying that each unit increase in neutrophil count, the expected hazard (death) of COVID-19 patients in ICU increase by $5.3 \%$. Figure 1 shows more illustrations in the forest plot of independent clinical variables predicting death in COVID-19 patients admitted to the ICU.

\section{Discussion}

In this study, we utilized the clinical and laboratory data of severe COVID-19 patients admitted in the ICU from five hospitals in Saudi Arabia to predict their potential outcomes. Our findings displayed a high mortality rate of $58.1 \%$ for our COVID-19 patients admitted to the ICU. A report from the United States showed that the death rate of COVID-19 patients in the ICU was approximately $67 \%$ in Washington, ${ }^{12}$ compared to $32 \%$ in the United Kingdom. ${ }^{13}$ According to a systematic review done in May 2020, the overall ICU mortality rate of COVID-19 patients was $25.7 \% .{ }^{14}$ In China, the overall ICU mortality rate was around $38 \%$, and interestingly very few of those patients had a chance to be mechanically ventilated. This difference seems to be due to a larger sample size as reports from China that had a sample size similar to ours had mortality rates of up to $61.5 \%{ }^{15}$ Moreover, $77 / 86$ $(89.5 \%)$ of our patients required mechanical ventilation and a study from Wuhan shown that $97 \%$ of patients who required invasive mechanical ventilation died. ${ }^{16}$ Shortness of breath was present in about $85 \%$ of our patients, which is in concordance with a meta-analysis that proved dyspnea in the COVID-19 patients admitted to the ICU was six times more than in the non-ICU group.${ }^{17}$ Our study revealed that patients who died were older than the survivors and that is consistent with findings from all over the world. ${ }^{18,19}$ Age appears to be a major risk factor that predicts progression of COVID-19 patients to ARDS and need for ICU admission and the incidence of complications in the geriatric population are considerably high, with more systemic complications. ${ }^{20}$ Survivors in our cohort had a higher cough symptom rate compared to the ones that succumbed to death $(100 \%$ vs $78 \% ; \mathrm{p}=$ 0.015 ), which suggests that presenting with cough may be more associated with moderate COVID-19 severity or severe COVID-19 with other features associated with a better survival outcome. ${ }^{21}$ Although a review of multiple 
Table 4 Significant Correlation Between Laboratory Variables Findings

\begin{tabular}{|c|c|c|c|}
\hline Variable I & Variable 2 & Correlation & Strength \\
\hline WBC Count & Neutrophil count & $0.718 * *$ & Moderate \\
\hline WBC Count & Platelet & $0.544 * *$ & Fair \\
\hline WBC Count & D-dimer & $0.570 * *$ & Fair \\
\hline WBC Count & CRP & $0.426 * *$ & Fair \\
\hline WBC Count & BUN & $0.27 I^{*}$ & Poor \\
\hline WBC Count & NLR Ratio & $0.599 * *$ & Fair \\
\hline Hemoglobin & BUN & $-0.262^{*}$ & Poor \\
\hline Hemoglobin & Ferritin & $0.232 *$ & Poor \\
\hline Neutrophil count & Platelet & $0.438 * *$ & Fair \\
\hline Neutrophil count & D-dimer & $0.609 * *$ & Moderate \\
\hline Neutrophil count & CRP & $0.373 * *$ & Fair \\
\hline Neutrophil count & ALT & $0.278 *$ & Poor \\
\hline Neutrophil count & BUN & $0.248 *$ & Poor \\
\hline Neutrophil count & Creatinine & $0.242 *$ & Poor \\
\hline Neutrophil count & NLR Ratio & $0.646 * *$ & Moderate \\
\hline Lymphocyte Count & Total Bilirubin & $-0.266^{*}$ & Poor \\
\hline Lymphocyte Count & NLR Ratio & $-0.550 * *$ & Fair \\
\hline Platelet & CRP & $0.279 *$ & Poor \\
\hline Platelet & NLR Ratio & $0.298 *$ & Poor \\
\hline D-dimer & CRP & $0.334 * *$ & Fair \\
\hline D-dimer & $\mathrm{LDH}$ & $0.347 * *$ & Fair \\
\hline D-dimer & BUN & $0.407 * *$ & Fair \\
\hline D-dimer & Creatinine & $0.272 *$ & Poor \\
\hline D-dimer & NLR Ratio & $0.377 * *$ & Fair \\
\hline CRP & $\mathrm{LDH}$ & $0.260^{*}$ & Poor \\
\hline CRP & NLR Ratio & $0.400 * *$ & Fair \\
\hline LDH & AST & $0.357 * *$ & Fair \\
\hline LDH & Creatinine & $0.254 *$ & Poor \\
\hline ALT & AST & $0.598 * *$ & Moderate \\
\hline ALT & Ferritin & $0.452 * *$ & Fair \\
\hline AST & Ferritin & $0.407 * *$ & Fair \\
\hline Creatinine & BUN & $0.714 * *$ & Moderate \\
\hline
\end{tabular}

Notes: Data were expressed as count (\%) unless otherwise. *P value less than 0.01 significant level. **P value less than 0.05 significant level.

Abbreviations: WBC, white blood cell; CRP, C-reactive protein; $L D H$, lactate dehydrogenase; ALT, alanine transaminase; AST, aspartate transaminase; BUN, blood urea nitrogen; NLR, neutrophil-lymphocyte ratio.

studies had illustrated that cough was associated with severe disease (OR 1.63, 95\% CI 1.03-2.60), it was not linked with ICU admission. ${ }^{17}$ However, since cough and fever are the most common presenting symptoms of COVID- $19,{ }^{22}$ it is difficult to infer and relate these symptoms to the patient's management outcomes. Despite the fact that there have been large advancements in researching sepsis throughout the years, sepsis is as yet one of the main causes of mortality in ICUs. ${ }^{22,23}$ Reports from the United States reveal that in nearly $70 \%$ of their COVID-19 patients, septic shock was severe enough to necessitate medications to support the heart and circulation. ${ }^{12,24}$ Our study indicates that $84 \%$ of patients who died suffered from septic shock $(\mathrm{p}<0.001)$ and that septic shock was indicative of expected death in COVID-19 patients admitted in the ICU $(\mathrm{OR}=58.1, \mathrm{HR}=9.5)$. Unfortunately, viral sepsis caused by SARS-CoV-2 has a high rate of being missed and thus daily evaluation of sepsis-related Sequential Organ Failure Assessment (SOFA) score and Multiple Organ Dysfunction (MOD) score is essential for early diagnosis, treatment, and prevention of septic shock and multi-organ dysfunction consequences in COVID-19 patients admitted in the ICU. ${ }^{25}$ Similarly, hypogammaglobulinemia that leads to bacterial pneumonia may also be missed during the chaotic situation of COVID-19. ${ }^{26}$ Regarding complications, AKI and ARDS were associated with grave outcomes in our patients $(\mathrm{p}<0.001)$. Research has shown that COVID-19 causes exuberant lung inflammation and can result in ARDS and respiratory failure. ARDS is usually more prevalent in severe COVID-19 patients and this may be due to the presence of higher levels of interleukin-6 (IL-6) and tissue necrosis factor-alpha (TNF- $\alpha){ }^{27}$ The elevated levels of cytokine or hypercytokinemia leads to ARDS along with multiple organ damage. ${ }^{28}$ Although an article had corroborated the advantage of using prednisone in COVID-19 patients, ${ }^{19}$ utilizing high-dose corticosteroids in critically ill patients is complicated and must be used with extreme care to avoid aggravating complications by worsening laboratory parameters and risk of death. ${ }^{29,30}$ Our findings revealed that AKI was an indicator of severe infection and death (OR (95\% CI): 7.279 (1.191-65.43), p $=0.032)$. Literature reports that the Asian population had the lowest pooled incidence rate of AKI at $5.5 \% .^{31}$ Recently, 12 hospitals in New York reported that an estimated $27 \%$ of their admitted patients had AKI, however, death among the AKI group was not reported. ${ }^{32}$ Our analysis suggested a significant moderate correlation between WBC count vs neutrophil count, neutrophil count vs D-dimer, neutrophil count vs NLR Ratio, ALT vs AST, and creatinine vs BUN. In our study, neutrophil count (HR (95\% CI): 1.053 (1.023-1.085), p < 0.001) was shown to predict clinical severity in ICU patients with COVID-19, this finding agrees with a systematic review that proven this laboratory parameter was feasible and cost-effective predictor for mortality among COVID-19 patients in ICU. ${ }^{33}$ Our patient sample was from the ICU, which is a high mortality group, thus neutrophil-lymphocyte ratio (NLR), CRP, ferritin, and inflammatory markers readings in this study could be highly biased towards a higher value. This could explain why the proven high 
Table 5 Logistic Regression Model for Death Among COVID-19 Patients in ICU

\begin{tabular}{|c|c|c|c|c|}
\hline \multirow[t]{2}{*}{ Predictors } & \multicolumn{2}{|l|}{ Unadjusted } & \multicolumn{2}{|l|}{ Adjusted } \\
\hline & Odds Ratio (Cl 95\%) & $P$ & Odds Ratio (Cl 95\%) & $P$ \\
\hline \multicolumn{5}{|l|}{ Control } \\
\hline Gender & $2.586(0.516-25.613)$ & 0.269 & $0.988(0.914-1.052)$ & 0.716 \\
\hline Age & $1.04 \mid(1.007-1.08)$ & 0.017 & $6.653(0.577-114.634)$ & 0.128 \\
\hline \multicolumn{5}{|l|}{ Interested } \\
\hline Cough & $0.073(0.00 \mathrm{I}-0.608)$ & 0.010 & $0.1(0-5.039)$ & 0.234 \\
\hline Septic Shock & 235 (27.8-309/9.2) & $<0.001$ & 58.1 (5.97-78|2.8) & $<0.001$ \\
\hline ARDS & $10.639(2.6-61.064)$ & $<0.001$ & $0.513(0.048-4.318)$ & 0.532 \\
\hline AKI & I 2.037 (3.9-44.572) & $<0.001$ & $7.279(1.191-65.43)$ & 0.032 \\
\hline Neutrophil & $1.13(1.008-1.319)$ & 0.009 & I (0.934-I.I49) & 0.995 \\
\hline D-dimer & $2.141(1.233-4.63)$ & 0.002 & $1.104(0.39-2.136)$ & 0.737 \\
\hline BUN & $1.223(1.026-1.554)$ & 0.014 & $0.934(0.80 I-1.465)$ & 0.398 \\
\hline Blood Culture & $9.4(1.086-1235.421)$ & 0.040 & $0.195(0-2503.576)$ & 0.588 \\
\hline
\end{tabular}

Note: $A \mathrm{p}$ value of $<0.05$ was considered significant.

Abbreviations: BUN, blood urea nitrogen; ARDS, acute respiratory distress syndrome; AKI, acute kidney injury.

Table 6 Cox Regression Model for Death Among COVID-19 Patients in ICU

\begin{tabular}{|c|c|c|c|c|}
\hline \multirow[t]{2}{*}{ Predictors } & \multicolumn{2}{|l|}{ Unadjusted } & \multicolumn{2}{|l|}{ Adjusted } \\
\hline & Hazard Ratio (Cl 95\%) & $\boldsymbol{P}$ & Hazard Ratio (Cl 95\%) & $\boldsymbol{P}$ \\
\hline \multicolumn{5}{|l|}{ Control } \\
\hline Gender & $1.304(0.585-2.903)$ & 0.516 & $0.976(0.95 I-I)$ & 0.054 \\
\hline Age & $1.016(0.998-1.033)$ & 0.082 & $1.894(0.75-4.78)$ & 0.176 \\
\hline \multicolumn{5}{|l|}{ Interested } \\
\hline Cough & $0.338(0.172-0.665)$ & 0.002 & $0.969(0.38 I-2.463)$ & 0.948 \\
\hline Septic Shock & I3.64 (5.832-31.89) & $<0.001$ & $9.502(2.958-30.524)$ & $<0.001$ \\
\hline ARDS & 6.191 (1.50I-25.54) & 0.012 & $1.2(0.226-6.364)$ & 0.831 \\
\hline AKI & 2.931 (1.534-5.602) & 0.001 & $2.059(0.64 I-6.6 I 2)$ & 0.225 \\
\hline Neutrophil & $1.058(1.036-1.079)$ & $<0.001$ & $1.053(1.023-1.085)$ & $<0.001$ \\
\hline D-dimer & $1.438(1.252-1.651)$ & $<0.001$ & $1.015(0.84 \mid-1.225)$ & 0.876 \\
\hline BUN & $1.048(1.015-1.082)$ & 0.004 & $1.009(0.966-1.053)$ & 0.699 \\
\hline Blood Culture & $14.33(5.8 \mid 6-35.3)$ & $<0.001$ & $1.642(0.247-10.902)$ & 0.608 \\
\hline
\end{tabular}

Note: A p-value of $<0.05$ was considered significant.

Abbreviations: BUN, blood urea nitrogen; ARDS, acute respiratory distress syndrome; AKI, acute kidney injury.

NLR, CRP, and ferritin levels did not significantly discriminate survival versus death of our COVID-19 patients in the ICU. Other studies that found these markers to be predictive of worsening disease had included mild and moderate COVID-19 cases admitted to the hospital in their sample of analysis and had a mortality rate of about $8-15 \%,{ }^{18}$ compared to our mortality rate of about $60 \%$. Studies have exhibited a correlation of severe cases, thrombosis, mortality, and a longer ICU stay with elevations in inflammatory markers and coagulation markers in COVID-19 patients. $^{34}$ This is because the infection triggers the coagulation system and there is a correlation of these markers with the activation of the proinflammatory cytokine cascade. ${ }^{35}$ Multiple studies indicate that higher NLR values were noticed in the severe group on hospital admission, augmented exponentially compared to those in the mild or moderate group, and were connected with mortality. ${ }^{36-38}$ Ferritin has also proven to be a valuable marker for COVID-19 severity and it was discovered that elevated ferritin was linked with a longer viral clearance time. ${ }^{39}$ Increased levels of CRP have also been shown to be prognostic in assessing the severity of 


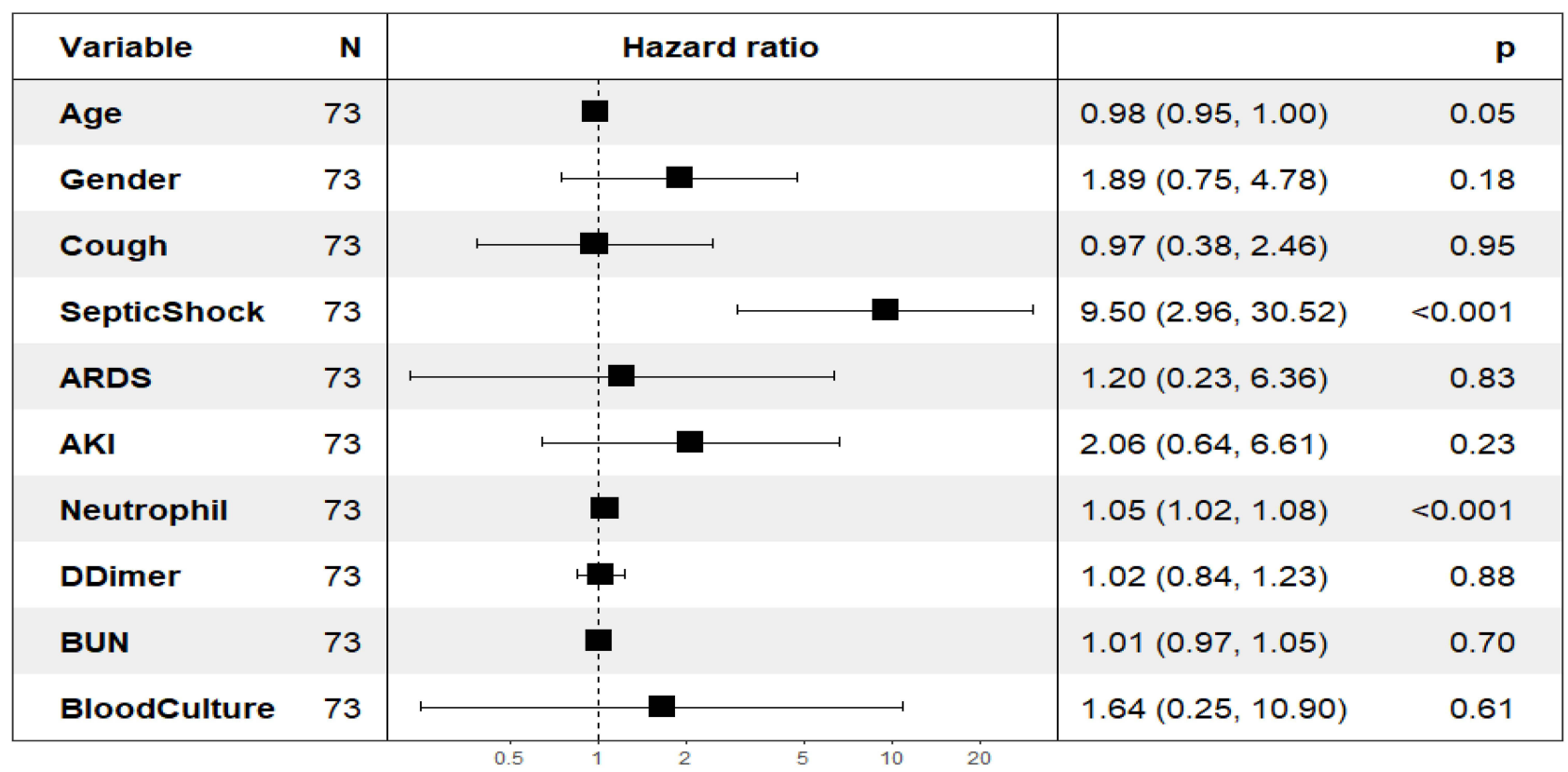

Figure I Forest plot of independent clinical variables predicting death in COVID-19 patients admitted to the ICU.

COVID-19. ${ }^{41,42}$ In a recent study by ${ }^{41}$ D-dimer levels were increased far out of proportion to any abnormalities in the PT/INR, activated PTT, fibrinogen level, or platelet count; these findings are uncharacteristic of DIC as currently understood. ${ }^{43}$ Understanding the pathogenesis of these mediators in COVID-19 may guide in developing targeted effective therapeutic interventions. ${ }^{44}$ These valuable predictive markers should be effectively monitored and appropriate interventions should be provided.

This study had several limitations. Firstly, this is shortterm cross-sectional retrospective observational study. A prospective study would give us better insight into the predictors of outcomes in severe COVID-19 patients. Secondly, some data were not in the case review form, eg, $\mathrm{PO}_{2} / \mathrm{FiO}_{2}$, which prevented us from including the APACHE II score in our analysis. We also did not study the impact of hypogammaglobulinemia on mortality of ICU admitted patients. Lastly, our sample size was small due to our emphasis on critically ill COVID-19 patients. We did not compare the severe patients with the mild to moderate severity patients. Collaboration with more national hospitals would lead to a larger cohort and a more accurate analysis.

\section{Conclusion}

This cross-sectional study investigated the impact of inflammatory markers and complications in the ICU on the outcomes of 73 severe COVID-19 patients in the Saudi population. Septic shock, AKI, and high neutrophil count were found to be predictive of death in these patients. Further research is needed from the Eastern Mediterranean region to aid efficient recognition and management of severe COVID-19 patients in our population.

\section{Ethical Approval}

This study was approved by the Institutional Review Board (IRB Log No. RC20.06.88-1) in Al Habib Research Center at Dr. Sulaiman Al-Habib Medical Group, Riyadh, Saudi Arabia.

\section{Consent}

All patient data was deidentified, and this study adhered to the ethical guidelines of the Declaration of Helsinki and good clinical practice.

\section{Acknowledgment}

The authors thank the referee for constructive comments.

\section{Author Contributions}

All authors made substantial contributions to conception and design, acquisition of data, or analysis and interpretation of data; took part in drafting the article or revising it critically for important intellectual content; agreed to submit to the current journal; gave final approval of the version to be published; and agree to be accountable for all aspects of the work. 


\section{Funding}

This research did not receive any specific grant from funding agencies in the public, commercial, or not-forprofit sectors.

\section{Disclosure}

The authors declare no conflicts of interest for this work.

\section{References}

1. Lu R, Zhao X, Li J, et al. Genomic characterisation and epidemiology of 2019 novel coronavirus: implications for virus origins and receptor binding. Lancet. 2020;395(10224):565-574. doi:10.1016/S0140-6736 (20)30251-8

2. World Health Organization. Coronavirus disease (COVID-19): weekly epidemiological update; 2020. Cited May 31, 2020.

3. Rabaan AA, Al-Ahmed SH, Al-Malkey MK, et al. Airborne transmission of SARS-CoV-2 is the dominant route of transmission: droplets and aerosols. Infez Med. 2021;29:10-19.

4. World Health Organization. WHO Coronavirus Disease (COVID-19) Dashboard; 2020. Available from: https:/covid19.who.int/table. Accessed August 16, 2020.

5. Ministry of Health. COVID 19 Dashboard: saudi Arabia; 2020. Available from: https://covid19.moh.gov.sa/. Accessed May 31, 2020.

6. Al Mutair A, Alhumaid S, Alhuqbani WN, et al. Clinical, epidemiological, and laboratory characteristics of mild-to-moderate COVID19 patients in Saudi Arabia: an observational cohort study. Eur J Med Res. 2020;25(1):1-8. doi:10.1186/s40001-020-00462-x

7. Alhumaid S, Al Mutair A, Al Alawi Z, et al. Clinical features and prognostic factors of intensive and non-intensive 1014 COVID-19 patients: an experience cohort from Alahsa, Saudi Arabia. Eur J Med Res. 2021;26(47). doi:10.1186/s40001-021-00517-7

8. Huang C, Wang Y, Li X, et al. Clinical features of patients infected with 2019 novel coronavirus in Wuhan, China. lancet. 2020;395 (10223):497-506. doi:10.1016/S0140-6736(20)30183-5

9. Zheng Y, Xu H, Yang M, et al. Epidemiological characteristics and clinical features of 32 critical and 67 noncritical cases of COVID-19 in Chengdu. $J$ Clin Virol. 2020;127:104366. doi:10.1016/j. jcv.2020.104366

10. Al-Omari A, Al Mutair A, Al Ammary M, Aljamaan F. A Multicenter Case-Historical Control Study on Short-Term Outcomes of TeleIntensive Care Unit. Telemed e-Health. 2020;26(5):645-650. doi:10.1089/tmj.2019.0042

11. Saudi Ministry of Health. ICU Triage, Admission, and Discharge Criteria during the COVID 19 pandemic V2 2020; 2021. Available from: https://www.moh.gov.sa/Ministry/MediaCenter/Publications/ Documents/ICU-Criteria-during.pdf. Accessed May 20, 2021.

12. Chan YH. Biostatistics 104: correlational analysis. Singapore Med J. 2003;44(12):614-619.

13. Wang X. Firth logistic regression for rare variant association tests. Front Genet. 2014;5:187. doi:10.3389/fgene.2014.00187

14. Firth D. Bias reduction of maximum likelihood estimates. Biometrika. 1993;80(1):27-38. doi:10.1093/biomet/80.1.27

15. Heinze G, Schemper M. A solution to the problem of separation in logistic regression. Stat Med. 2002;21(16):2409-2419. doi:10.1002/ sim. 1047

16. Arentz M, Yim E, Klaff L, et al. Characteristics and outcomes of 21 critically ill patients with COVID-19 in Washington State. JAMA 2020;323(16):1612-1614. doi:10.1001/jama.2020.4326

17. BBC News. A third of Covid-19 intensive care patients do not recover, study shows; 2020. Available from: https://www.bbc.com/ news/uk-scotland-52653192. Accessed July 9, 2021.
18. Quah P, Li A, Phua J. Mortality rates of patients with COVID-19 in the intensive care unit: a systematic review of the emerging literature. Critical Care. 2020;24:1-4.

19. Yang X, Yu Y, Xu J, et al. Clinical course and outcomes of critically ill patients with SARS-CoV-2 pneumonia in Wuhan, China: a singlecentered, retrospective, observational study. Lancet Respir Med. 2020;8.5:475-481. doi:10.1016/S2213-2600(20)30079-5

20. Rabaan AA, Al-Ahmed SH, Muhammad J, et al. Role of inflammatory cytokines in COVID-19 patients: a review on molecular mechanisms, immune functions, immunopathology and immunomodulatory drugs to counter cytokine storm. Vaccines. 2021;9(5):436. doi:10.3390/vaccines9050436

21. Zhou F, Yu T, Du R, et al. Clinical course and risk factors for mortality of adult inpatients with COVID-19 in Wuhan, China: a retrospective cohort study. lancet. 2020;395(10229):1054-1062. doi:10.1016/S0140-6736(20)30566-3

22. Jain V, Yuan JM. Predictive symptoms and comorbidities for severe COVID-19 and intensive care unit admission: a systematic review and meta-analysis. Int $J$ Public Health. 2020;65:533-546. doi:10.1007/s00038-020-01390-7

23. Wu Z, McGoogan JM. Characteristics of and important lessons from the coronavirus disease 2019 (COVID-19) outbreak in China: summary of a report of 72314 cases from the Chinese Center for Disease Control and Prevention. JAMA. 2020;323(13):1239-1242. doi:10.1001/jama.2020.2648

24. Wu C, Chen X, Cai Y, et al. Risk factors associated with acute respiratory distress syndrome and death in patients with coronavirus disease 2019 pneumonia in Wuhan, China. JAMA Intern Med. 2020;180(7):934-943. doi:10.1001/jamainternmed.2020.0994

25. Alsofayan YM, Althunayyan SM, Khan AA, Hakawi AM, Assiri AM. Clinical characteristics of COVID-19 in Saudi Arabia: a national retrospective study. $J$ Infect Public Health. 2020;13 (7):920-925. doi:10.1016/j.jiph.2020.05.026

26. Kempker JA, Martin GS. A global accounting of sepsis. Lancet. 2020;395(10219):168-170. doi:10.1016/S0140-6736(19)33065-X

27. Rudd KE, Johnson SC, Agesa KM, et al. Global, regional, and national sepsis incidence and mortality, 1990-2017: analysis for the Global Burden of Disease Study. Lancet. 2020;395(10219):200-211. doi:10.1016/S0140-6736(19)32989-7

28. Rabaan AA, Al-Ahmed SH, Garout MA, et al. Diverse Immunological Factors Influencing Pathogenesis in Patients with COVID-19: a Review on Viral Dissemination, Immunotherapeutic Options to Counter Cytokine Storm and Inflammatory Responses. Pathogens. 2021;10(5):565. doi:10.3390/pathogens 10050565

29. Bhatraju PK, Ghassemieh BJ, Nichols M, et al. Covid-19 in critically ill patients in the Seattle region-case series. New Engl J Med. 2020;382(21):2012-2022. doi:10.1056/NEJMoa2004500

30. Liu D, Wang Q, Zhang H, et al. Viral sepsis is a complication in patients with Novel Corona Virus Disease (COVID-19). Med Drug Discovery. 2020;8:100057. doi:10.1016/j.medidd.2020.100057

31. Alhumaid S, Al Mutair A, Al Alawi Z, et al. Coinfections with Bacteria, Fungi, and Respiratory Viruses in Patients with SARSCoV-2: A Systematic Review and Meta-Analysis. Pathogens. 2021;10(7):809.

32. Kobayashi T, Jung SM, Linton NM, et al. Communicating the risk of death from novel coronavirus disease (COVID-19). J Clin Med. 2020;9(2):580. doi:10.3390/jcm9020580.

33. Bossù $P$, Toppi E, Sterbini V, Spalletta G. Implication of aging related chronic neuroinflammation on COVID-19 pandemic. J Personalized Med. 2020;10(3):102. doi:10.3390/jpm10030102

34. Fu EL, Janse RJ, de Jong Y, et al. Acute kidney injury and kidney replacement therapy in COVID-19: a systematic review and metaanalysis. Clin Kidney J. 2020;13(4):550-563. doi:10.1093/ckj/sfaa160

35. Richardson S, Hirsch JS, Narasimhan M, et al. Presenting characteristics, comorbidities, and outcomes among 5700 patients hospitalized with COVID-19 in the New York City area. JAMA. 2020;323 (20):2052-2059. doi:10.1001/jama.2020.6775 
36. Wang G, Wu C, Zhang Q, et al. C-reactive protein level may predict the risk of COVID-19 aggravation. In: Open Forum Infectious Diseases. US: Oxford University Press; 2020:ofaa153.

37. Cheng Y, Luo R, Wang K, et al. Kidney disease is associated with inhospital death of patients with COVID-19. Kidney Int. 2020;97 (5):829-838. doi:10.1016/j.kint.2020.03.005

38. Lagunas-Rangel FA. Neutrophil-to-lymphocyte ratio and lymphocyte-to-C-reactive protein ratio in patients with severe coronavirus disease 2019 (COVID-19): a meta-analysis. J Med Virol. 2020;92 (10):1733-1734. doi:10.1002/jmv.25819

39. Shorr AF, Thomas SJ, Alkins SA, Fitzpatrick TM, Ling GS. D-dimer correlates with proinflammatory cytokine levels and outcomes in critically ill patients. Chest. 2002;121(4):1262-1268. doi:10.1378/ chest.121.4.1262

40. Yan X, Li F, Wang X, et al. Neutrophil to lymphocyte ratio as prognostic and predictive factor in patients with coronavirus disease 2019: a retrospective cross-sectional study. J Med Virol. 2020;92 (11):2573-2581. doi:10.1002/jmv.26061
41. Cao P, Wu Y, Wu S, et al. Elevated serum ferritin level effectively discriminates severity illness and liver injury of coronavirus disease 2019 pneumonia. Biomarkers. 2020;26(3):207-212.

42. Tan C, Huang Y, Shi F, et al. C-reactive protein correlates with computed tomographic findings and predicts severe COVID-19 early. J Med Virol. 2020;92(7):856-862. doi:10.1002/jmv.25871

43. Upadhyay J, Tiwari N, Ansari MN. Role of inflammatory markers in corona virus disease (COVID-19) patients: a review. Exp Biol Med. 2020;245(15):1368-1375. doi:10.1177/1535370220939477

44. Al-Samkari H, Karp Leaf RS, Dzik WH, et al. COVID-19 and coagulation: bleeding and thrombotic manifestations of SARS-CoV2 infection. Blood, J Am Society Hematol. 2020;136(4):489-500.

\section{Publish your work in this journal}

The International Journal of General Medicine is an international, peer-reviewed open-access journal that focuses on general and internal medicine, pathogenesis, epidemiology, diagnosis, monitoring and treatment protocols. The journal is characterized by the rapid reporting of reviews, original research and clinical studies across all disease areas. The manuscript management system is completely online and includes a very quick and fair peer-review system, which is all easy to use. Visit http://www.dovepress.com/ testimonials.php to read real quotes from published authors. 